\title{
Spontaneous recovery after extinction with periodic time-outs'
}

\begin{abstract}
Three pigeons were trained on a variable-interval schedule. Following this, they were extinguished. During extinction, 1-min. periods of time-in (TI) and time-out (TO) alternated. The Ss responded only in TI. When extinction was complete, TO was removed in the middle of a session. All Ss begain responding shortly after the removal of TO.
\end{abstract}

\section{Introduetion}

Skinner's (1950) discussion of spontaneous recovery provides an alternative to the inhibitory mechanism proposed by Hull (1943). Briefly put, it is this: distinctive stimuli occur at the beginning of an experimental session and it takes repeated extinction in the presence of these stimuli to achieve complete extinction. These stimuli may be initiated by handling, being put into the apparatus, etc. If the animal is reinforced in the presence of stimuli initiated by such events, they may become discriminative and will increase the probability of a response until repeated extinction in their presence occurs.

From the above, it may be predicted that spontaneous recovery may be made to occur at any point in time by simply reinstating stimulus conditions in which extinction is not complete. This experiment tests this notion.

\section{Subjects and Apparatus}

Three homing pigeons were used. Two were experimentally sophisticated and one naive. The two sophisticated birds had histories of variable interval (No. 3) and variable ratio (No. 6). The remaining bird (No. 12) was trained especially for this study. Ss were run at about $80 \%$ of free-feeding weight.

The experiment was conducted in a one-key pigeon chamber of standard dimensions (Ferster \& Skinner, 1957). The key was horizontally centered 8 in from the floor. General illumination was provided by an overhead 60-w light. Grain reinforcement was provided for $3 \mathrm{sec}$. at appropriate times. Relay and timing circuitry were used to program the contingencies and responses were recorded on a cumulative recorder. The chamber and programming equipment were housed in separate rooms.

\section{Procedure}

The two sophisticated Ss were placed directly on a variable interval schedule with a mean of $1 \mathrm{~min}$. (VI1). The naive $S$ was placed on this schedule after preliminary key training. During all VI training, a yellow light illuminated the response key. Each $\mathrm{S}$ was exposed to the VI schedule for $1 \mathrm{hr}$. a day for about two weeks. This was extended to about three weeks for No.12, the naive
S. After VI training, extinction was initiated. During extinction Ss were given 2-hr. sessions. In extinction, Ss were exposed to alternating 1 -min periods of time-in (TI) and time-out (TO). TI consisted of exactly the same conditions that prevailed during VI, with the exception that reinforcement was omitted. During TO the chamber was in total darkness. Pigeons do not respond during such a TO (Ferster \& Skinner, 1957).

When extinction during TI was complete, tests for spontaneous recovery were conducted. The extinction criterion used was three sessions without any responding during TI. This amounted to $3 \mathrm{hr}$. of exposure to TI. Spontaneous recovery tests were conducted simply by removing TO and allowing the $\mathrm{S}$ to remain in TI. Spontaneous recovery tests were not initiated until about $45 \mathrm{~min}$. after the beginning of the session. If spontaneous recovery occurred, the test was usually terminated after about 3-5 min. Each $\mathrm{S}$ was given at least three tests on three different occasions. One $S$ (No. 3) was allowed to respond for approximately an hr. during his third test.

\section{Results}

Figures 1 and 2 present typical results. Two spontaneous recovery tests are presented for each of two Ss, No. 3 and No. 6 . The offset marker on the pen represents TO while the pen in the up position represents TI. Shortly after the last TO No. 3 makes a few responses; after a pause of about a min., he begins to respond again and emits about 500 responses before the test is

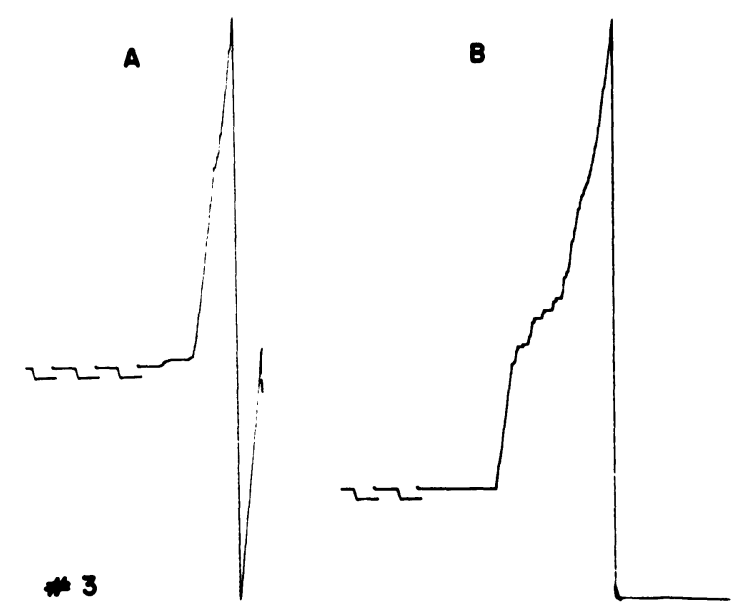

Fig. 1. Sample cumulative records of two spontaneous recovery tests for pigeon No. 3 . 


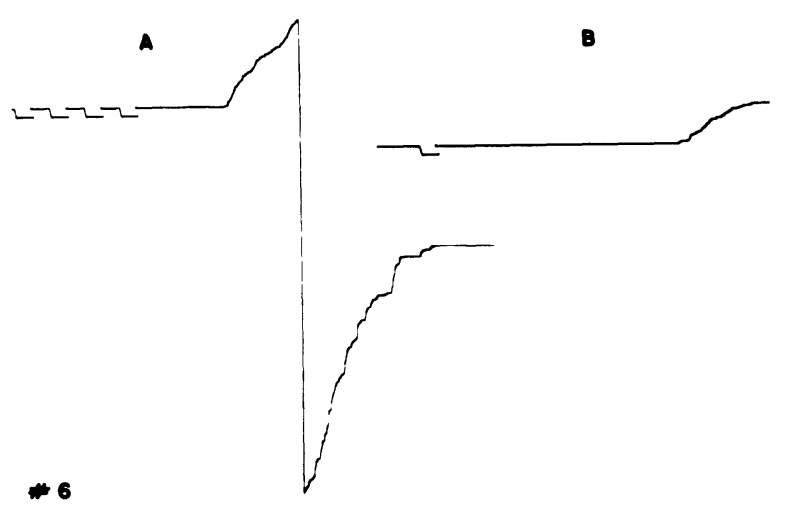

Fig. 2. Sample cumulative records of two spontaneous recovery tests for pigeon No. 12 .

terminated. During the second test, this $\mathrm{S}$ pauses for about 5 min. after the last TO. After responding begins he emits about 400 responses before the test is terminated. During the third test (not shown) he was allowed to respond for about an hr. during which he emitted over 2700 responses. Similar results are presented for No. 6. No record for No. 12 is presented here, but it displays spontaneous recovery after an initial pause of about 25 min. after the last TO (first test).

It may be concluded that under the conditions of this experiment, spontaneous recovery may be produced at times other than at the beginning of a session.

\section{Diseussion}

The interpretation of the above results assumes that the TO initiates stimuli which are correlated with the passage of time (Anger, 1963). TO was used in this experiment in place of stimuli associated with the beginning of the session as in Skinner's (1950) discussion. During extinction the $\mathrm{S}$ is extinguished only after TO; that is, during the stimuli initiated by TO. When these stimuli have dissipated, the $S$ experiences stimuli in the presence of which he is not extinguished.

In terms of Skinner's discussion, this experiment is analogous to giving repeated extinction only for a short while after the beginning of a session. When the $\mathrm{S}$ is given a session the same duration as sessions during which he was reinforced, we would expect spontaneous recovery later in the session.

It may be argued that the phenomenon observed in this experiment is not true spontaneous recovery since VI training and extinction occurred under different conditions. It may be noted, however, that while the $\mathrm{S}$ was actually emitting extinction responses, the environment was, in fact, exactly the same. Consider, for instance, an experiment where $2 \mathrm{hr}$. separated successive TOs during extinction. In this case the $\mathrm{S}$ would be exposed to the same conditions during VI and extinction and spontaneous recovery would not be expected to occur after the terminal TO.

\section{References}

ANGER, D. The role of temporal discriminations in the reinforcement of Sidman avoidance behavior. J. exp. Anal. Behav., 1963, 6, 477-506.

FERSTER, C. B., \& SKINNER, B. F. Schedules of reinforcement. New York: Appleton-Century-Crofts, 1957.

HULL, C. L. Principles of behavior. New York: Appleton-CenturyCrofts, 1943.

SKINNER, B. F. Are theories of learning necessary? Psychol. Rev., $1950,57,193-216$.

Note

1. This experiment was conducted at Emory University and reported at the 1962 meeting of the Southeastern Psychological Association. 